\title{
On optimal geodetic network design for fault-mechanics studies
}

\author{
M. D. Gerasimenko ${ }^{1}$, N. V. Shestakov ${ }^{1}$, and Teruyuki Kato ${ }^{2}$ \\ ${ }^{1}$ Institute of Applied Mathematics, 690041 Vladivostok, Russia \\ ${ }^{2}$ Earthquake Research Institute, University of Tokyo, Tokyo, Japan
}

(Received October 21, 1999; Revised August 10, 2000; Accepted August 22, 2000)

\begin{abstract}
This paper deals with the optimal design of GPS geodynamic geodetic networks. A simple strike-slip fault-model is used to investigate the design of networks and to decide where to place geodetic stations in order to satisfy the accuracy of fault-model parameter estimates, which sites should be selected from the initial mass of permissible points which are bounded by topography and other conditions.
\end{abstract}

\section{Introduction}

As is well-known, with the development of advanced positioning techniques especially such as the Global Positioning System (GPS) in the last two decades, the interest to the optimal design of the monitoring schemes for investigation of the deformation of earth's crust and behavior of individual fault strand has grown. A brief review the purposes and present state of the art of network optimization for the last one is depicted by Johnson and Wyatt (1994). The main purposes of geodetic network optimization are concerned with designing a geodetic survey to achieve some desired level of accuracy in positioning, high reliability and low cost (Schmitt, 1985; Kuang, 1991; etc.). However, it should be noted that the problem of geodetic network design was not fully solved. There is no analytical method that solves the problem of how many stations to include in the network. Nor is there a method that provides guidance as to which sites should be chosen to provide the most information about fault mechanics or deformation on a nearby fault. The initial mass of permissible points are bounded by topography and other conditions such as the cost at erecting of monuments, driving, setting up the instruments, etc. Excluding the papers Gerasimenko (1990, 1991, 1997), no previous works has been done towards the solving of this problem. It is for reason that this report was intended to solve this problem in a few degrees and indicate the way for future research to optimize our understanding of fault mechanics.

\section{Formulation and Solution of the Optimal Design Problem}

Required precision, reliability and economy generally characterize the quality of a geodetic network. Different from the previous publications, this report deals with the integer-programming problem how many stations should be included into the network and which sites should be chosen to optimize our understanding of fault mechanics. To facilitate the discussion, this report concentrates on a simplest

Copy right (C) The Society of Geomagnetism and Earth, Planetary and Space Sciences (SGEPSS); The Seismological Society of Japan; The Volcanological Society of Japan; The Geodetic Society of Japan; The Japanese Society for Planetary Sciences. strike-slip fault model in which the displacement parallel to the fault is (Lisowski et al., 1991; Johnson and Wyatt, 1994; etc.)

$$
d(x)=-\frac{V}{\pi} \arctan \left(\frac{x}{H}\right)
$$

where $x$ is the distance perpendicular to the fault. The fault plane extends from the surface of the half-space to infinite depth, locked from the surface to $H \mathrm{~km}$, and freely slipping below this depth at $V$ meter per year. Uniform motion $V$ of two blocks relative to a fixed reference frame is positive for right-lateral motion. Figure 1 show expected surface deformation as a function of distance from the fault trace.

Model (1) is a one-dimensional two-parameter standard strike-slip model of dislocation theory. The fault is long and straight and deformation is uniform in the direction of fault strike.

Such type of model is acceptable for example, to the San Andreas fault system. We will assume that the spacetechnique is able to determine fault parallel displacements $d(x)$ of the points, which resides on two blocks, with a standard deviation of $m_{d}$. We can include to the value $m_{d}$ the monument instability and another type of errors as well. It is especially important because it acts to mask the tectonic signals of interest.

From the practical point of view it is obviously that geodetic network can be realized in a most economical way. That is why the first most important criterion to the network is the cost requirement. A common cost criterion for optimization can be stated as

$$
\Phi=\sum_{1}^{n} c_{i} p_{i} \rightarrow \min
$$

where

$$
p_{i}=\left(\begin{array}{ccc}
\frac{1}{m_{d_{i}}^{2}} & \text { or } & 0
\end{array}\right)
$$

is the weight of observation and $c_{i}$ is the suitably chosen coefficient of profitableness for observable which take into account the erecting of geodetic monument, driving to the station, etc. Zero optimal weights $p_{i}=0$ mean that the corresponding observables do not contribute to the accuracy of 


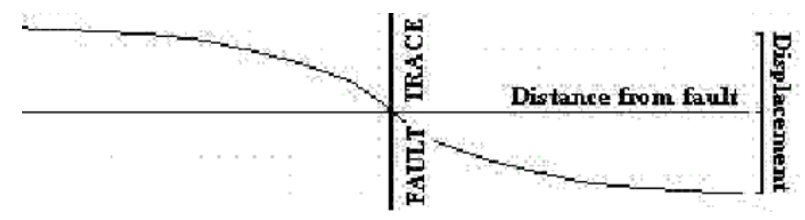

Fig. 1. Expected surface deformation as a function of distance from the fault trace.

fault parameters to be investigated and therefor these observables should be deleted from the final observational scheme.

To facilitate the solution of integer problem we change the condition (3) by constraint

$$
0 \leq p_{i} \leq p_{i, \max }
$$

where

$$
p_{i, \max }=\frac{1}{m_{d_{i}}^{2}}
$$

is the maximum accessible weight. The constraint equation (4) means that the weights to be optimally solved for should be non-negative and achievable with the given instrumentation.

Instead of target function (2) we can choose as well

$$
\Phi_{1}=\sum_{1}^{n} \frac{1}{c_{i} p_{i}} \rightarrow \max
$$

but this criterion is not equivalent to the criterion (2) in mathematical sense. Moreover, it is not recommended because numerical problem of solving will occur: when individual weight $p_{i} \rightarrow 0$, the function $\Phi_{1} \rightarrow \infty$.

Another most important criteria of the network are the precision requirements. We can express its by constraint equations for the precision of slip-rate

$$
m_{V}^{2} \leq m_{V, \max }^{2}
$$

and precision of locking-depth

$$
m_{H}^{2} \leq m_{H, \max }^{2}
$$

Inequality constraints expressed by Eqs. (7)-(8) are applied to ensure that resulting accuracy $m_{V}$ and $m_{H}$ of the parameters $V$ and $H$ are better or equal a certain boundary values accuracy $m_{V, \text { max }}$ and $m_{H, \max }$. The values $m_{V}^{2}=Q_{V V}$ and $m_{H}^{2}=Q_{H H}$ are the diagonal elements of the covariance matrix

$$
Q=\left(\begin{array}{ll}
Q_{V V} & Q_{V H} \\
Q_{H V} & Q_{H H}
\end{array}\right)=\left(\begin{array}{cc}
\sum p_{i} a_{i} a_{i} & \sum p_{i} a_{i} b_{i} \\
\sum p_{i} a_{i} b_{i} & \sum p_{i} b_{i} b_{i}
\end{array}\right)^{-1} .
$$

The coefficients $a_{i}$ and $b_{i}$ comes from linearization of the model (1) as

$$
\delta d\left(x_{i}\right)=a_{i} \delta V+b_{i} \delta H
$$

where

$$
\begin{aligned}
& a_{i}=\frac{\partial d\left(x_{i}\right)}{\partial V}=-\frac{1}{\pi} \operatorname{arctg}\left(\frac{x_{i}}{H}\right), \\
& b_{i}=\frac{\partial d\left(x_{i}\right)}{\partial H}=\frac{V}{\pi} \frac{x_{i}}{H^{2}+x_{i}^{2}} .
\end{aligned}
$$

If we take into consideration the more complicated model of the fault than the model (1) with more than two parameters (see, for example, Matsu'ura et al., 1986), we can include to a number of constraints the precision requirements to these parameters. For simplicity we discuss here only precision criteria. Other types of design criteria such as reliability or the total number of redundant observables can be easily incorporated to obtain a multi-objective optimal design. The interested reader is referred to Gerasimenko (1990), Kuang (1991), etc.

As a result at above mathematical model, a non-linear programming problem can be formulated as

$$
\begin{aligned}
& \Phi=\sum_{1}^{n} c_{i} p_{i} \rightarrow \min \\
& 0 \leq p_{i} \leq p_{i, \max } \\
& m_{V}^{2} \leq m_{V, \max }^{2} \\
& m_{H}^{2} \leq m_{H, \max }^{2}
\end{aligned}
$$

This non-linear optimization problem can be solved by well-known mathematical methods. We have used for this purpose a gradient method with a penalty functions (see, for example, Gerasimenko, 1992). It should be noted that an iterative solution to the above mathematical model should be applied since Taylor series of linear form is being used. The iteration process stops when the solution ceases to change.

A FORTRAN-77 computer program has been written to implement in practice the developed methodology (it is available by request from the first author by e-mail: mdger@iammail.febras.ru). The input information includes the coordinates $x$ of the initial mass of permissible points as obtained from a field reconnaissance, the assumed values of fault parameters $V$ and $H$ with theirs necessary precision, and achievable accuracy of displacements to be measured. The output information includes the optimal observational weights for all observation points. Zero or close to zero optimal weights mean that these points do not contribute to the accuracy of the fault parameters and should be deleted from the final observational scheme.

To demonstrate the practical applications of the developed optimization modeling, a few simplest numerical examples are provided below. In all examples the parameter $c_{i}=1$, $i=1,2, \ldots, n$.

\section{Numerical Examples}

Example 1. Suppose, that 80 geodetic stations can be equally spaced within $40 \mathrm{~km}$ of the fault trace over $1 \mathrm{~km}$ each other. The fault-model parameters are chosen: the slip-rate is taken to be $30 \mathrm{~mm} / \mathrm{yr}$ with precision not less than $m_{V \text {, max }}=2$ $\mathrm{mm} / \mathrm{yr}$, and the locking-depth $H=10 \mathrm{~km}$ with $m_{H, \max }=2$ $\mathrm{km}$. The measurement technique is able to determine faultparallel displacements relative to a fixed reference frame with a standard deviation $m_{d}=1 \mathrm{~mm} / \mathrm{yr}$.

The result of optimization is (see Fig. 2): only 8 geodetic stations must be fixed. 4 stations should be located in the distance farthest from the fault trace, i.e. $x= \pm 40 \mathrm{~km}$ and $x= \pm 37 \mathrm{~km} ; 4$ stations should be located in the points with $x= \pm 7 \mathrm{~km}$ and $x= \pm 8 \mathrm{~km}$.

Example 2. Different from the Example 1, here a points can be fixed only on one side of the fault trace. That is why 


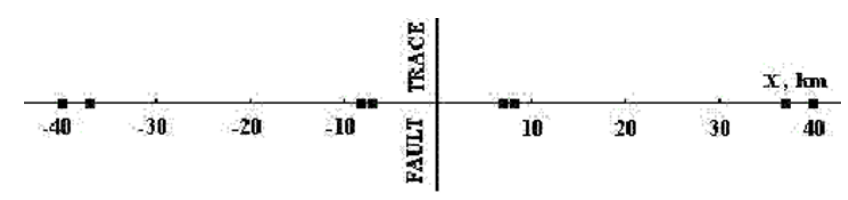

Fig. 2. Optimal station locations. From 80 geodetic stations were selected only 8 stations contributing to accuracy of the fault parameters.

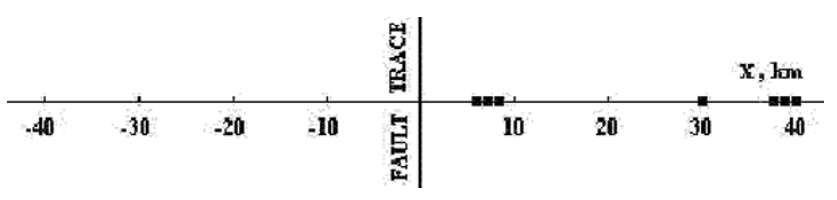

Fig. 3. Optimal station locations. Here points were fixed on one side of the fault trace and initial number of permissible points equal 40. After optimization were selected only 7 station.

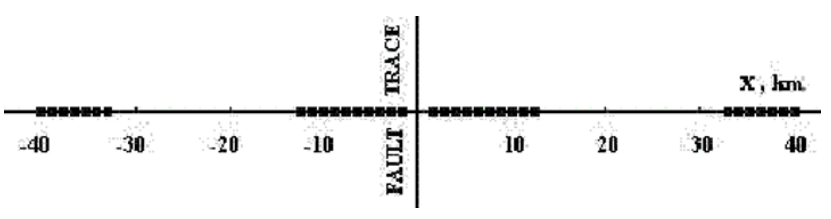

Fig. 4. Optimal station locations. The input information is the same as in Example 1, but slip-rate $V=10 \mathrm{~mm} / \mathrm{yr}$ and $m_{H}=2500 \mathrm{~m}$. From 80 initial point's mass were selected only 36 geodetic stations.

the initial number of permissible points equal 40. Another input information is the same as in Example 1.

The result of optimization is (see Fig. 3): we must fix geodetic stations in the points with coordinates $x$ equal 6,7 , 8, 30, 38, 39 and $40 \mathrm{~km}$.

Example 3. The input information is the same as in Example 1 but the slip-rate $V=10 \mathrm{~mm} / \mathrm{yr}$. The result of optimization is: all points being only provided a standard deviation $m_{H}=2200 \mathrm{~m}$ even though a value $m_{V}=0.8 \mathrm{~mm}$.

If we take as input $m_{H \text {, max }}=2500 \mathrm{~m}$, the result is (see Fig. 4): 34 stations must be fixed in the all points with coordinates $x$ from $\pm 3 \mathrm{~km}$ to $\pm 12 \mathrm{~km}$ and from $\pm 34 \mathrm{~km}$ to \pm 40 $\mathrm{km}$. The value $m_{V}=1 \mathrm{~mm}$.

\section{Conclusion}

As noted by Johnson and Wyatt (1994), it is the most difficult question of where best to locate GPS observing sites. They investigated the situation when 20 geodetic stations were evenly spaced. As well they applied the simulated annealing algorithm proceeds toward the preferred station locations for the case when the number of stations in the network are fixed, but each geodetic station is allowed to move from one iteration to the next one. We do not know anyone previous investigation in this direction.

In this report we described the main idea of a more practical method for the optimal design of geodetic monitoring schemes in order to estimate the fault-model parameters, sliprate and locking-depth, as accurately as possible. The results of optimization enable us to make decision which sites should be selected from the hundreds of available points and where they should be located in order to estimate the unknown parameters and achieve the desired criteria of precision. Also the numerical examples concludes that points at $\sim 0.75$ and $\sim 4.0$ fault depths are most useful. The smaller is a slip-rate the more points must be included to the network especially a nearby fault as well. These results confirms what many investigators would guess. The given examples demonstrated clearly the beauty of and the benefits one may get from the developed methodology.

Acknowledgments. We thank W. H. Prescott (USGS) and anonymous reviewer for their critical reviews of the manuscript which made the text more readable.

\section{References}

Gerasimenko, M. D., About problems of optimal design of geodetic networks, in State and Outlook of Engineering Geodetic and Photogrammetric Works, pp. 11-15, TsNIIGAiK, Moscow, 1990 (in Russian).

Gerasimenko, M. D., First order design of geodetic networks, Geodesy and Cartography, No. 5, 4-7, 1991 (in Russian).

Gerasimenko, M. D., Optimal Design and Adjustment of Geodetic Networks, Nauka, Moscow, 160 pp., 1992 (in Russian).

Gerasimenko, M. D., First order design of the deformation networks with the minimal number of geodetic points and their optimal allocation, Far Eastern Mathematical Reports, 4, 86-94, 1997.

Johnson, H. O. and F. K. Wyatt, Geodetic network design for fault-mechanics studies, Manuscripta Geodaetica, 19, 309-323, 1994.

Kuang, S. L., Optimization and Design of Deformation Monitoring Scheme, Ph.D. dissertation, Dept. of Surveying Engineering Technical Report No. 157, University of New Brunswick, 179 pp., Fredericton, Canada, 1991.

Lisowski, M., J. C. Savage, and W. H. Prescott, The Velocity field along the San Andreas fault in Central and Southern California, J. Geophys. Res., 96(B5), 8369-8389, 1991.

Matsu'ura, M., D. D. Jackson, and A. Cheng, Dislocation model for a seismic Crystal Deformation at Hollister, California, J. Geophys. Res., 91(B12), 12661-12674, 1986.

Schmitt, G., Review of Network Design: Criteria, Risk Functions, Design Ordering, in Optimization and Design of Geodetic Network, edited by E. Grafarend and F. Sanco, Springer, Berlin etc., pp. 6-10, 1985.

M. D. Gerasimenko (e-mail: mdger@iam-mail.febras.ru), N. V. Shestakov, and T. Kato (e-mail: teru@eri.u-tokyo.ac.jp) 